\title{
Multiplex ligation-dependent probe amplification identifies copy number changes in normal and undetectable karyotype MDS patients
}

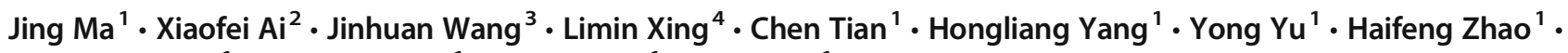 \\ Xiaofang Wang ${ }^{1} \cdot$ Zhigang Zhao $^{1} \cdot$ Yafei Wang $^{1} \cdot$ Zeng Cao $^{1}$ (D)
}

Received: 19 November 2020 / Accepted: 1 May 2021 / Published online: 15 May 2021

(C) The Author(s) 2021

\begin{abstract}
Chromosomal abnormalities play an important role in classification and prognostication of myelodysplastic syndrome (MDS) patients. However, more than 50\% of low-risk MDS patients harbor a normal karyotype. Recently, multiplex ligation-dependent probe amplification (MLPA) has emerged as an effective and robust method for the detection of cytogenetic aberrations in MDS patients. To characterize the subset of MDS with normal karyotype or failed chromosome banding analysis, we analyzed 144 patient samples with normal karyotype or undetectable through regular chromosome banding analysis, which were subjected to parallel comparison via fluorescence in situ hybridization (FISH) and MLPA. MLPA identifies copy number changes in $16.7 \%$ of 144 MDS patients, and we observed a significant difference in overall survival (OS) (median OS: undefined vs 27 months, $\mathrm{p}=0.0071$ ) in patients with normal karyotype proved by MLPA versus aberrant karyotype cohort as determined by MLPA. Interestingly, patients with undetectable karyotype via regular chromosome banding indicated inferior outcome. Collectively, MDS patients with normal or undetectable karyotype via chromosome banding analysis can be further clarified by MLPA, providing more prognostic information that benefit for individualized therapy.
\end{abstract}

Keywords Myelodysplastic syndromes · Cytogenetic analysis · Multiplex ligation-dependent probe amplification; Normal karyotype $\cdot$ Undetectable chromosome pattern

\section{Introduction}

Myelodysplastic syndrome (MDS) is a heterogeneous group of hematologic neoplasms classically described as a clonal

Jing Ma, Xiaofei Ai and Jinhuan Wang contributed equally to this work.

Zhigang Zhao

goodluckzho001@163.com

Yafei Wang

drwang2005@163.com

Zeng Cao

caozeng1@ sina.com

1 Department of Hematology and Blood and Marrow Transplantation, Tianjin Medical University Cancer Institute and Hospital, National Clinical Research Center for Cancer, Tianjin's Clinical Research Center for Cancer, Key Laboratory of Cancer Prevention and Therapy, Huan-Hu-Xi Road, Ti-Yuan-Bei, Hexi District, Tianjin 300060, China disorder of hematopoietic stem cells leading to dysplasia and ineffective hematopoiesis in the bone marrow [1]. Chromosomal abnormalities play an important role in classification and prognostication of MDS patients; however, more
2 Department of Pathology, Institute of Hematology and Blood Diseases Hospital, Chinese Academy of Medical Sciences \& Peking Union Medical College, No. 288 Nanjing Road, Heping District, Tianjin 300020, China

3 Department of Oncology, The Second Hospital of Tianjin Medical University, No.23 Pingjiang Road, Hexi District, Tianjin 300211, China

4 Hematology Department of General Hospital, Tianjin Medical University, No.154 Anshan Road, Heping District, Tianjin 300052, China 
than $50 \%$ of low-risk MDS patients harbor a normal karyotype as revealed by regular chromosome banding analysis $[2$, 3]. While chromosome banding analysis can only detect gains and/or losses of more than $10 \mathrm{Mb}$ size, it depends on proliferation of the MDS clone to obtain metaphases in vitro. Therefore, it is necessary to find a more targeted, highthroughput, simple, and cost-effective method with higher resolution and accuracy targeting at the clinically relevant lesions which have been described in MDS patients.

Multiplex ligation-dependent probe amplification (MLPA) assay is a technique for copy number variation (CNV) identification in many human genes simultaneously. So far, over 300 probe sets specific for a very large range of genetic disorders are commercially available. MLPA is a multiplex polymerase chain reaction (PCR)-based technique that can quantify up to 50 different genomic targets simultaneously in a single experiment through amplification of specific hybridizing probes [4-7]. One of the major advantages is the high specificity, because it can distinguish sequences differing in length by only one nucleotide. Another advantage is the low amount of input DNA (minimum of 20-50 ng) required for a successful MLPA reaction [8]. In this study, we addressed the question whether MDS patients with normal or without result after banding cytogenetics harbors cytogenetically cryptic gains or losses could be detected by MLPA.

\section{Methods}

\section{Patients and samples}

A total of 258 patients from January 2008 to December 2018 were retrospectively enrolled and were diagnosed with MDS according to the World Health Organization (WHO) Criterion 2016. Bone marrow samples at initial diagnosis of these patients were obtained. Among these patients, 144/258 (55.8\%) cases showed either normal karyotype $(\mathrm{n}=132 ; 51.2 \%)$ or without result after banding cytogenetics $(n=12 ; 4.6 \%)$. The male to female ratio was $1.48: 1$, and the median age of the patients was 53 years old (range: $15-83$ years old) with the median follow-up time of 18 months (range: 2-101 months). Patients were given the informed consent to genetic analyses and laboratory data for research studies. The study was approved by the Medical Ethics Committee of the Tianjin Cancer Institute and Hospital.

\section{Cytogenetic analysis}

Cytogenetic studies using standard G-banding techniques on heparinized BM samples were performed as part of the diagnostic work-up. At least 20 metaphase cells were analyzed whenever possible. Clonal abnormalities were defined as 2 or more cells with the same chromosomal gain or structural rearrangement or at least 3 cells with the same chromosomal deletion. Chromosome identification and karyotype descriptors used the International System for Human Cytogenetic Nomenclature (ISCN) [9].

\section{Interphase fluorescence in situ hybridization (FISH)}

FISH was performed on whole bone marrow mononuclear cells. In brief, commercial, multicolor probes provided by Vysis laboratories (Abbott Laboratories, IL, USA) were used that included probes for $-5 /-5 q,-7 /-7 q,+8,-20 q$, and $17 p-$, respectively. The following probes were used: LSI (locusspecific identifier) EGR1/D5S23, D5S721 Dual Color Probe for chromosome 5q; LSI D7S522/CEP 7 Probe for chromosome 7q; CEP (centromere enumeration probe) 8 DNA Probe for chromosome 8; LSI D20S108 Probe for chromosome 20q; and TP53 Probe for chromosome 17; all probes were obtained from Vysis Inc. (Downers Grove, IL, USA). The evaluation of FISH signals was performed using a fluorescence microscope (Olympus BX51, Japan) equipped with Q-FISH imaging software (IMSTAR, France). We counted 200-300 interphase nuclei for each slide. The positive threshold percentages were established according to previously published recommendations. The FISH results were interpreted in each institution by at least two experienced molecular pathologists, independent of concurrent metaphase karyotyping. Results were described according to the ISCN criteria [9].

\section{Multiplex ligation-dependent probe amplification}

Bone marrow specimens were collected from patients at diagnosis. Genomic DNA was extracted using the AxyPrep Blood Genomic DNA Miniprep Kit (Axygen Biosciences, cat no. AP-MN-BL-GDNA-250 Union city, CA, USA). Fifty nanograms of gDNA were subjected to MLPA analysis by using SALSA MLPA P414-A1 MDS probe mix (MRC-Holland, Amsterdam, Netherlands). The probe mix contained 46 probes targeting at chromosomal regions of interest in MDS and 12 internal reference gene probes targeting at regions that are generally unchanged in MDS. MLPA reactions including internal quality controls and negative controls were performed according to the manufacturer instructions. The PCR products were analyzed using ABI 3130XL Genetic analyzer (Applied Biosystems, Foster City, CA, USA) and Coffalyser.net software (MRC Holland, Amsterdam, Netherlands) according to the manufacture instruction. In addition, 10 DNA samples derived from the peripheral blood of healthy donors were subjected to MLPA analysis. The "Mean \pm 2 SD" (95\% CI, $\mathrm{P}=0.05$ ) and "Mean \pm 3 SD" ( $95 \% \mathrm{CI}, \mathrm{P}=0.01$ ) values for each individual probe are listed in our raw data. To improve the evaluation of the results with a larger CI, the "Mean \pm 3 SD" reference range was used as the cutoff value for CNV determination in our study. 


\section{Statistical analysis}

Survival curves were plotted by the Kaplan-Meier method, and the difference was assessed by log-rank test. Overall survival (OS) was measured from the time of diagnosis to the date of death or last follow-up. The statistically significant difference was considered at $\mathrm{p}<0.05$.

\section{Results}

\section{Characterization of patients with normal karyotype or no result after banding cytogenetics}

As shown in Fig. 1, normal karyotype and cases without result after banding cytogenetics are approximately 55.8\%. MLPA identifies copy number changes in 24 (16.7\%, 24/144 ) patients. Among these 24 patients, 10 patients showed chromosome banding analysis failed. For patients with normal karyotype, $10.6 \%$ (14/132) were identified with CNVs. Characteristics of 144 patients are shown in Table 1. The 144 MDS patients were divided into four subgroups based on MLPA and karyotype results; there were 86 males and 58 females. According to the classification of WHO 2016 version, the most common subtype is MDS-MLD. We calculated the Revised International Prognostic Scoring System scores (IPSS-R) and confirmed that 8 patients were very high risk, 28 patients with high risk, 48 patients with intermediate risk, 50 patients with low risk, and 10 patients with very low-risk disease.

\section{Abnormalities detected by MLPA in 14 MDS patients with normal karyotype}

Among 24 patients, 14 patients showed normal karyotype, which are shown in Table 2. According to cytomorphology, the cohort comprised the following MDS subtypes: MDS-RS $(\mathrm{n}=1)$, MDS-MLD ( $\mathrm{n}=6)$, MDS-EB-1 $(\mathrm{n}=4)$, and MDS-EB-2 $(\mathrm{n}=3)$. The most common CNV was -17p (P53-4b, TP53-1).

Chromosome 8 abnormality was positive in 1 case $(7.1 \%, 1 / 14)$; it showed $8 \mathrm{q}$ amplification. Chromosome 5 abnormality was positive in 1 case $(7.1 \%, 1 / 14)$. Chromosome 7 abnormality was positive in 1 case $(7.1 \%, 1 / 14)$. Chromosome 20 abnormalities were positive in 3 cases $(21.3 \%, 3 / 14)$. Chromosome 17 abnormalities including both $17 \mathrm{p}$ and $17 \mathrm{q}$ deletions were positive in 6 cases $(42.9 \%, 6 / 14), 5$ patients for $17 p$ deletion, and 1 patient for $17 \mathrm{q}$ deletion. Chromosome 11 abnormalities were positive in 2 cases $(14.2 \%, 2 / 14)$, and both showed $11 \mathrm{q}$ amplifications. One patient showed chromosome19 abnormalities including both $19 p$ and $19 q$ deletions. All detected aberrations are summarized in Table 2.

\section{Abnormalities detected by MLPA in 10 MDS patients without result after banding cytogenetics}

Among 24 patients, 10 patients with no result after banding cytogenetics are shown in Table 3 . The cohort involved the following MDS subtypes: MDS-RS ( $n=1)$, MDS -MLD ( $n=4)$, MDS-EB-1 $(n=2)$, MDS-EB-2 $(n=1)$, and $\operatorname{MDS}-\mathrm{U}(\mathrm{n}=2)$. The most common CNVs were -7 and +8 .

Chromosome 8 abnormalities including both $8 \mathrm{p}$ and $8 \mathrm{q}$ amplifications were positive in 4 cases $(40 \%, 1 / 10)$. Chromosome 5 abnormalities were positive in 2 cases (20\%, 2/10). Chromosome 7 abnormalities including both $7 \mathrm{q}$ deletion and $7 \mathrm{p}$ deletion were positive in 4 cases $(40 \%$, $4 / 10$ ), with two patients including both $7 p$ and $7 q$ deletions. Chromosome 20 abnormalities were not detected. Chromosome 17 abnormality was positive in 1 case
Fig. 1 Diagnostic approach in 258 MDS patients. Distribution of patients with aberrant karyotype, normal cytogenetics, and failed chromosome banding analysis is depicted

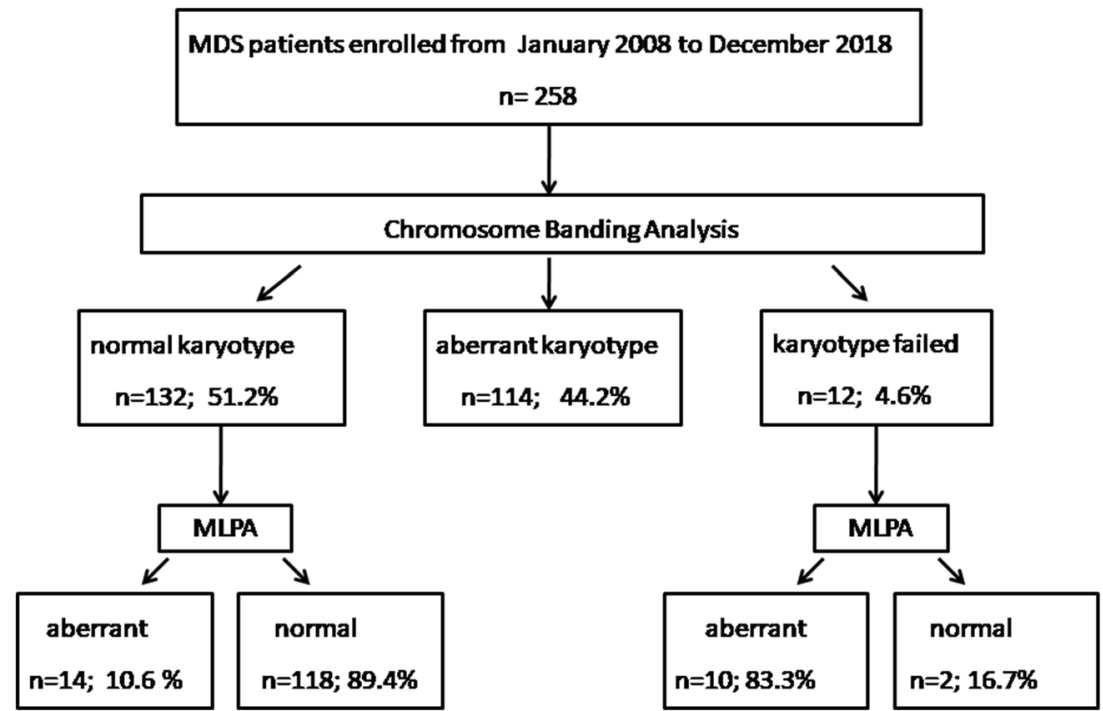


Table 1 Characteristics of MDS patients in our study ( $n=144)$

\begin{tabular}{|c|c|c|c|c|c|}
\hline & Total n (\%) & $\begin{array}{l}\text { Normal karyotype } \\
\text { and MLPA+ }\end{array}$ & $\begin{array}{l}\text { Normal karyotype } \\
\text { and MLPA- }\end{array}$ & $\begin{array}{l}\text { Failed karyotype } \\
\text { and MLPA+ }\end{array}$ & $\begin{array}{l}\text { Failed karyotype } \\
\text { and MLPA- }\end{array}$ \\
\hline N. of patients & $144(100 \%)$ & 14 & 118 & 10 & 2 \\
\hline Median age (years) & 53 & 47.5 & 57 & 44 & 51 \\
\hline \multicolumn{6}{|l|}{ Sex } \\
\hline Male & $86(59.7 \%)$ & 13 & 66 & 6 & 1 \\
\hline Median Hgb (g/L) & 76 & 72 & 81 & 76 & 79 \\
\hline Median ANC $\left(\times 10^{9} / \mathrm{L}\right)$ & 1.2 & 1.0 & 1.1 & 0.9 & 1.2 \\
\hline Median platelet count $\left(\times 10^{9} / \mathrm{L}\right)$ & 72 & 89 & 76 & 64 & 70 \\
\hline \multicolumn{6}{|l|}{ WHO 2016} \\
\hline MDS-SLD & $1(0.7 \%)$ & 0 & 1 & 0 & 0 \\
\hline MDS-RS & $6(4.2 \%)$ & 1 & 4 & 1 & 0 \\
\hline MDS-MLD & $68(47.2 \%)$ & 6 & 56 & 4 & 2 \\
\hline MDS-EB-1 & $33(22.9 \%)$ & 4 & 27 & 2 & 0 \\
\hline MDS-EB-2 & $29(20.1 \%)$ & 3 & 25 & 1 & 0 \\
\hline MDS-U & $7(4.9 \%)$ & 0 & 5 & 2 & 0 \\
\hline \multicolumn{6}{|l|}{ IPSS-R risk category } \\
\hline Very low & $10(7.0 \%)$ & 2 & 8 & 0 & 0 \\
\hline Low & $50(34.7 \%)$ & 6 & 42 & 2 & 0 \\
\hline Intermediate & $48(33.3 \%)$ & 1 & 46 & 1 & 0 \\
\hline High & $28(19.4 \%)$ & 4 & 16 & 6 & 2 \\
\hline Very high & $8(5.6 \%)$ & 1 & 6 & 1 & 0 \\
\hline
\end{tabular}

(10\%, 1/10). Chromosome11 abnormality was positive in 1 case $(10 \%, 1 / 10)$. One patient showed chromosome 19 abnormalities including both $19 \mathrm{p}$ and $19 \mathrm{q}$ deletions. All detected aberrations are summarized in Table 3.

\section{Comparison of MLPA assay and FISH}

To evaluate the performance of MLPA as a candidate method for the identification of CNVs in MDS patients, five

Table 2 Copy number changes identified by MLPA in 14 MDS patients with normal karyotype

\begin{tabular}{|c|c|c|c|c|c|}
\hline Case & Age & Gender & Diagnosis & Karyotype based on MLPA & $\begin{array}{l}\text { Assumed karyotype } \\
\text { according to MLPA }\end{array}$ \\
\hline Patient 1 & 69 & Male & MDS-EB-1 & 11q(4): KMT2A-4,KMT2A-36,TIRAP-3,ETS1-10 & $+11 \mathrm{q}$ \\
\hline Patient 2 & 72 & Male & MDS-RS & 11q(4): KMT2A-4,KMT2A-36,TIRAP-3,ETS1-10 & $+11 \mathrm{q}$ \\
\hline Patient 3 & 50 & Male & MDS-MLD & 20q(1): ASXL1-4 & $-20 q$ \\
\hline Patient 4 & 63 & Male & MDS-EB-2 & 8q(3): NCOA2-5,MYC-3,PTK2-33 17p(2): TP53-4b,TP53-1 & $+8 \mathrm{q}-17 \mathrm{p}$ \\
\hline Patient 9 & 39 & Male & MDS-MLD & $17 p(2):$ TP53-4b,TP53-1 & $-17 \mathrm{p}$ \\
\hline Patient 10 & 36 & Male & MDS-MLD & 17p(3): TP53-10,TP53-4b,TP53-1 & $-17 p$ \\
\hline Patient 11 & 46 & Male & MDS-EB-2 & $17 p(2):$ TP53-4b,TP53-1 & $-17 p$ \\
\hline Patient 12 & 51 & Male & MDS-MLD & 11q(1): KMT2A-4(+) 17q(1): NF1-17 SUZ12-12(+) & $-17 p$ \\
\hline Patient 13 & 42 & Male & MDS-EB-1 & $17 q(1): N F 1-17$ & $-17 q$ \\
\hline Patient 14 & 34 & Female & MDS-EB-2 & 19p(1):SMARCA4-25 19q(1): PRPF31-14 & -19 \\
\hline Patient 15 & 32 & Male & MDS-MLD & 20q(2):MMP9-9,ZMYND8-14 & $-20 q$ \\
\hline Patient 16 & 57 & Male & MDS-EB-1 & 20q(3): ASXL1-4,SRC-6,ZMYND8-14 & $-20 q$ \\
\hline Patient 19 & 47 & Female & MDS-MLD & 5q(3):APC-18,EGR1-1,EGR1-2 & $-5 q$ \\
\hline Patient 24 & 61 & Female & MDS-EB-1 & 7q(4): CDK6-8 SAMD9L-5,MLL5-4,MET-13 & $-7 q$ \\
\hline
\end{tabular}




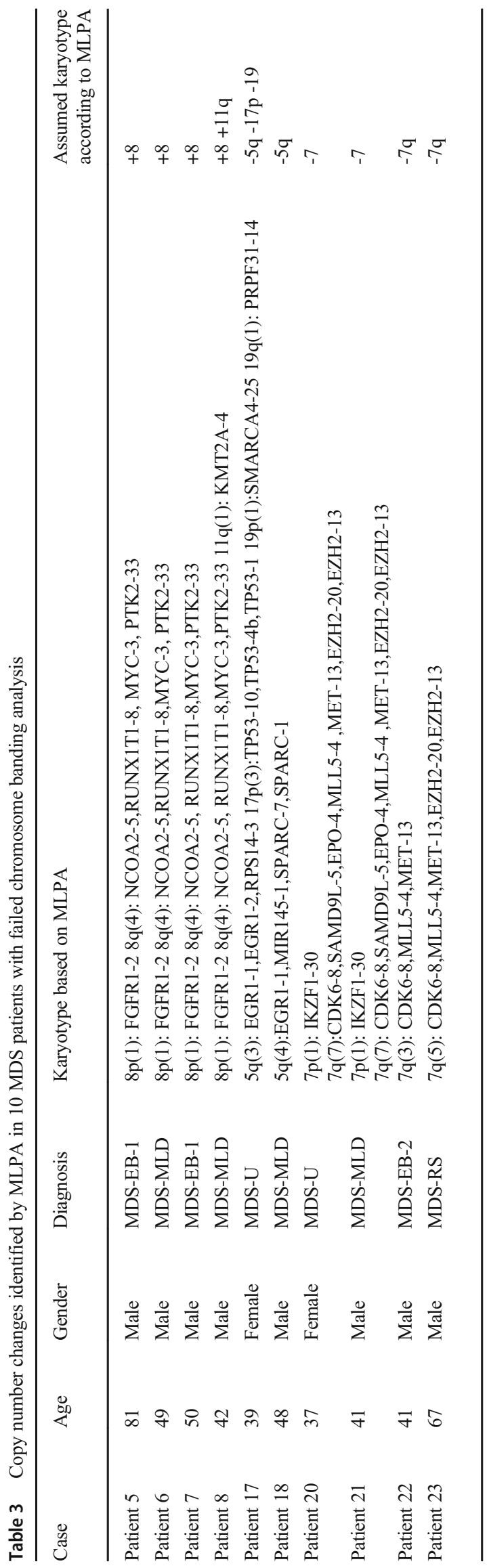

abnormalities, including $-5 /-5 \mathrm{q},-7 /-7 \mathrm{q},+8,-20 \mathrm{q}$, and $17 \mathrm{p}-$, were detected by FISH and MLPA. FISH results of 144 cases were compared with that of MLPA. Among 144 MDS patients, 137 results were concordant, and the whole consistency was $95.1 \%$. The genetic lesions determined by FISH and MLPA are listed in Table 4. Using MLPA analysis, clonal cytogenetic abnormalities were detected in 24 MDS patients with normal and undetectable karyotype, and 19/24 (79.2\%) of those patients were reclassified into a higher-risk IPSS-R prognostic category. Using FISH, 62.5\% (15/24) of MDS patients showed chromosomal abnormalities, whereas MLPA analysis showed that $100 \%(24 / 24)$ of MDS cases contained at least one CNV. Patient 8 and patient 17 showed two CNVs and three CNVs of MLPA analysis, respectively. All the additional detected aberrations by MLPA are summarized in Table 4.

\section{Survival analysis}

We performed survival analysis and compared the outcome of patients which were also confirmed by MLPA $(n=120)$ versus patients with aberrant karyotype as determined by MLPA $(n=24)$. We observed a significant difference in survival (median OS: undefined vs 27 months, $p=0.0071$, Fig. 2). We performed a survival analysis of normal karyotypes and cases without result after banding cytogenetics, respectively. Data were shown in Supplement Fig.1. We can see that there was no significant difference $(\mathrm{p}=0.1877, \mathrm{p}=0.2864)$ in the impact of MLPA results on OS of patients with normal karyotype and patients with failed karyotype. However, the curves of the two groups were clearly separated, and perhaps the difference was significant with the increase in the number of patients enrolled.

In addition, we compared the outcome of patients with normal karyotype $(n=132)$ to patients without result after banding cytogenetics $(n=12)$. It showed a significant difference in survival (median OS: undefined vs 26 months, $\mathrm{p}=0.0059$, Fig. 3 ), indicating that patients without result after banding cytogenetics had worse survival.

In our study, we also explored the impact of cytogenetic aberrations detected by MLPA on OS of lower-risk and higher-risk patients (defined according to IPSS-R) with a normal or without result after banding cytogenetics via Rbanding test. Lower-risk IPSS-R group included very lowrisk patients, low-risk patients, and intermediate patients with score $\leq 3.5$. Higher-risk IPSS-R group included intermediate patients with score $>3.5$, high risk, and very high-risk patients. For lower-risk IPSS-R patients (73/144), there were no differences in OS ( $p=0.5207$; Fig. $4 a)$. For higher-risk IPSS-R patients (71/144), OS was significantly shorter in the higher-risk patients with cytogenetic aberrations detected using MLPA $(n=19)$ compared with other higher-risk patients $(n=52)$ (median OS: 21 vs. undefined months, $p=0.0281$; Fig. 4b). 
Table 4 Genetic lesions determined by FISH and MLPA

\begin{tabular}{lllllll}
\hline Case & Diagnosis & IPSS-R risk & Karyotype & FISH & MLPA & IPSS-R risk group by MLPA \\
\hline Patient 5 & MDS-EB-1 & Very high & Failed & +8 & +8 & Very high \\
Patient 6 & MDS-MLD & High & Failed & +8 & +8 & Very high \\
Patient 7 & MDS-EB-1 & High & Failed & +8 & +8 & Very high \\
Patient 8 & MDS-MLD & High & Failed & +8 & $+8+11 \mathrm{q}$ & Very high \\
Patient 18 & MDS-MLD & High & Failed & $-5 \mathrm{q}$ & $-5 \mathrm{q}$ & High \\
Patient 20 & MDS-U & Intermediate & Failed & -7 & -7 & Very high \\
Patient 22 & MDS-EB-2 & High & Failed & $-7 \mathrm{q}$ & $-7 \mathrm{q}$ & Very high \\
Patient 23 & MDS-RS & Low & Failed & $-7 \mathrm{q}$ & $-7 \mathrm{q}$ & Very high \\
Patient 17 & MDS-U & Intermediate & Failed & $-5 \mathrm{q}$ & $-5 \mathrm{q}-17 \mathrm{p}-19$ & Very high \\
Patient 4 & MDS-EB-2 & Very high & Normal & $+8-17 \mathrm{p}$ & $+8-17 \mathrm{p}$ & Very high \\
Patient 9 & MDS-MLD & Low & Normal & $-17 \mathrm{p}$ & $-17 \mathrm{p}$ & High \\
Patient 10 & MDS-MLD & Low & Normal & $-17 \mathrm{p}$ & $-17 \mathrm{p}$ & High \\
Patient 15 & MDS-MLD & Low & Normal & $-20 \mathrm{q}$ & $-20 \mathrm{q}$ & Low \\
Patient 16 & MDS-EB-1 & Low & Normal & $-20 \mathrm{q}$ & $-20 \mathrm{q}$ & Intermediate \\
Patient 24 & MDS-EB-1 & High & Normal & $-7 \mathrm{q}$ & $-7 \mathrm{q}$ & Very high \\
Patient 21 & MDS-MLD & High & Failed & Negative* & -7 & Very high \\
Patient 1 & MDS-EB-1 & High & Normal & Negative & $+11 \mathrm{q}$ & High \\
Patient 2 & MDS-RS & High & Normal & Negative & $+11 \mathrm{q}$ & High \\
Patient 3 & MDS-MLD & Very low & Normal & Negative & $-20 \mathrm{q}$ & Low \\
Patient 11 & MDS-EB-2 & Low & Normal & Negative & $-17 \mathrm{p}$ & High \\
Patient 12 & MDS-MLD & Very low & Normal & Negative & $-17 \mathrm{p}$ & Low \\
Patient 13 & MDS-EB-1 & Intermediate & Normal & Negative & $-17 \mathrm{q}$ & Very high \\
Patient 14 & MDS-EB-2 & Low & Normal & Negative & -19 & Intermediate \\
Patient 19 & MDS-MLD & High & Normal & Negative & $-5 \mathrm{q}$ & Very high \\
\hline
\end{tabular}

*Negative just for $-5 /-5 \mathrm{q},-7 /-7 \mathrm{q},+8,-20 \mathrm{q}$, and $17 \mathrm{p}$ -

\section{Discussion}

In MDS, the IPSS-R classifiers have clearly showed the prognostic impact of distinct cytogenetic abnormalities; these cytogenetic abnormalities include -7/7q-, -5/5q-, +8, 20q-, -Y, i(17q) or $\mathrm{t}(17 \mathrm{p}),-13 / 13 \mathrm{q}-, 11 \mathrm{q}-, 12 \mathrm{p}-$, or $\mathrm{t}(12 \mathrm{p})$, and the most common abnormalities $\left(-7 / 7 q_{-},-5 / 5 q-,+8\right.$, and $\left.20 q_{-}\right)$occur in approximately $40 \%$ of MDS cases $[10,11]$. The majority of chromosome aberrations in MDS are gains and deletions of

\section{OS}

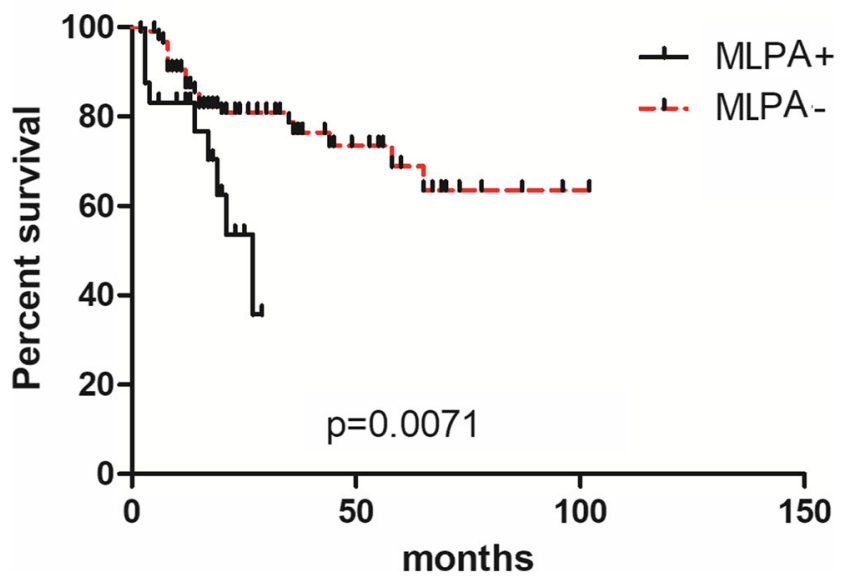

Fig. 2 OS analysis of patients harboring aberration $(n=24)$ or not detected $(\mathrm{n}=120)$ by MLPA in 144 MDS patients with normal and failed karyotype chromosomal material, while balanced rearrangements are rare [3]. As we all know, more than $50 \%$ of MDS showed a normal karyotype. For MDS patients with normal karyotype, FISH is increasingly used for cytogenetic analysis because of its higher resolution and greater success rate. Nevertheless, it is difficult for FISH to screen all lesions simultaneously due to the high cost and technique limitation. Although established as the golden standard for detection of chromosomal aberrations in MDS, karyotyping (which requires cell amplification) and FISH are low-resolution, time-consuming, labor-intensive, and costly assays compared to MLPA, while small probes are directed at

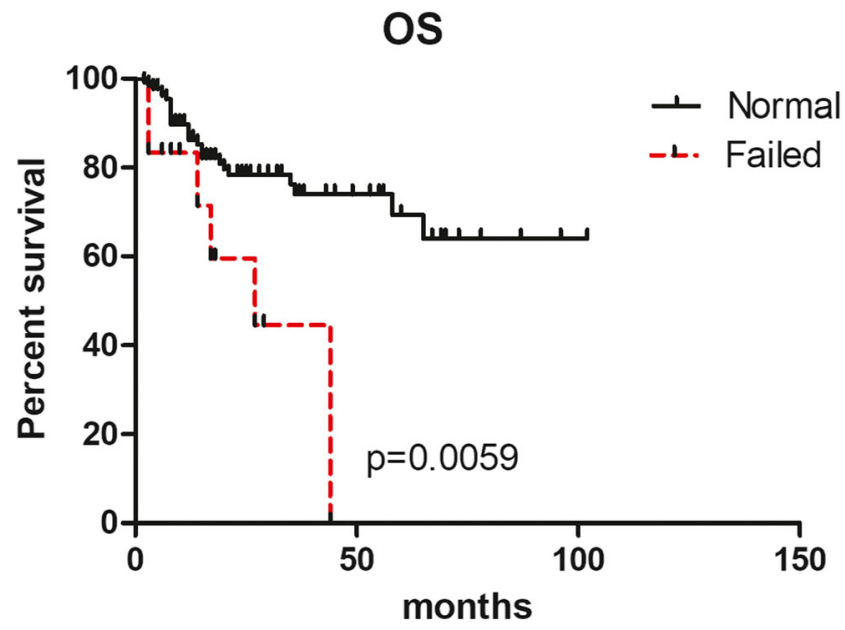

Fig. 3 OS of patients with normal karyotype $(n=132)$ and failed chromosome banding analysis $(n=12)$ 
Fig. 4 OS of patients with cytogenetic aberrations detected using MLPA compared with other patients in lower risk IPSSR group (a MLPA+:n=5, MLPA:n=68) and higher risk IPSS-R group (b MLPA+:n=19, MLPA$: \mathrm{n}=52)$
OS (lower-risk IPSS-R)

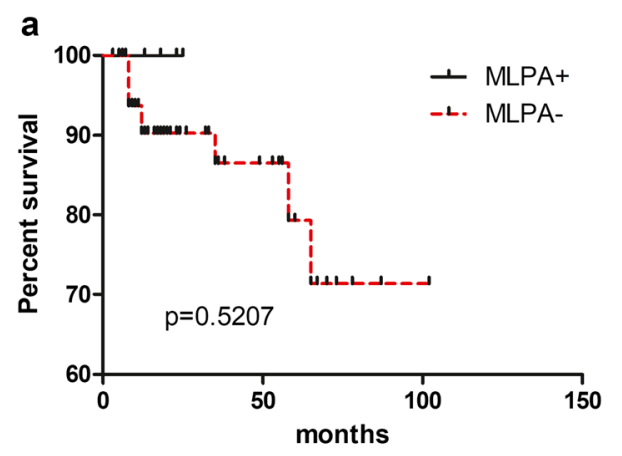

OS (higher-risk IPSS-R)

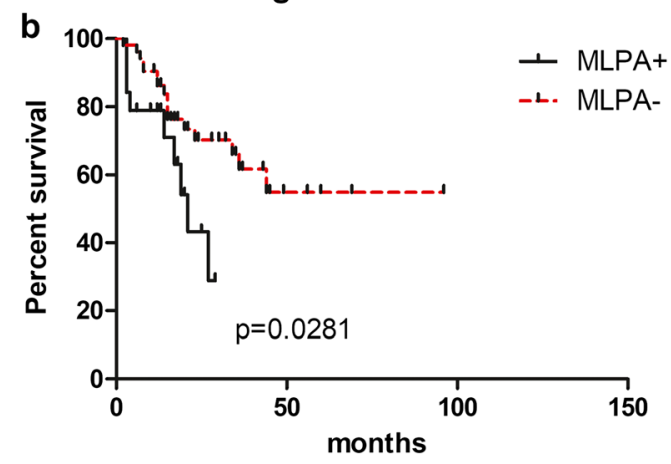

regions of interest in MDS, providing a resolution higher than that of FISH and bacterial artificial chromosome (BAC)-based array comparative genomic hybridization $(\mathrm{aCGH})$ and equivalent to oligo-based aCGH $[6,7]$. MLPA has a considerably higher resolution and can identify small unbalanced chromosomal aberrations undetectable by chromosome banding analysis.

Donahue et al. [12] showed that MLPA has higher accuracy and specificity than FISH in MDS and ALL patients. Array CGH and MLPA have been used as a method of choice for diagnosis of MDS patients with unexplained genetic aberrations. Volkert et al. [13] detected CNVs in $11 \%$ of 520 MDS patients with a normal karyotype using array CGH. Wang et al. [14] analyzed 437 MDS patients using an MLPA assay and detected clonal genetic abnormalities in $9.2 \%$ of cases with a normal or failed karyotype. In our study, we analyzed 258 MDS patients using MLPA assay and detected clonal genetic abnormalities in $16.7 \%$ of normal or failed karyotype patients. In our cohort, the proportion of patients with CNVs was higher, probably because of the higher proportion of patients with karyotype failure. For normal karyotype, MLPA detected clonal genetic abnormalities in $10.6 \%$ of 132 patients. Among 144 MDS patients, the consistency of FISH and MLPA was $95.1 \%$, and no patient showed cytogenetic abnormalities detected just by FISH. Our results provide evidence that MLPA has an advantage over FISH for MDS patients.

As we all know, chromosome banding analysis is very important for MDS. Unfortunately, a variety of issues hamper cytogenetic evaluation in cases because chromosome banding studies may be hindered by several factors, including low proliferative rate in tissue culture, insufficient number of metaphase cells, reduced cell viability or hypo cellularity upon arrival to the reference laboratory, poor chromosome morphology, or complexity of the karyotype $[15,16]$. So the outcomes of these patients were poorly understood. In our study, $4.6 \%$ of MDS patients showed failed banding cytogenetics, while we indicated that these patients may encounter a poor outcome as detected by MLPA (Fig. 3). On the other hand, the significance of failed banding cytogenetics on outcome of MDS patients has been scarcely reported. Medeiros et al. [17] compared the baseline characteristics and the prognostic impact of 94 (6\%) AML patients with failed banding cytogenetics to the remaining 1403 AML patients with successful karyotype.
These patients without result after banding cytogenetics had a lower response rate to induction chemotherapy, and the complete remission and survival rates were similar to those seen in patients with unfavorable karyotype. Lazarevic et al. [18] analyzed 1737 AML patients; the frequencies of unsuccessful cytogenetics and unperformed cytogenetics were $2.1 \%$ and $20 \%$, respectively. Their research showed that a lack of cytogenetic data translates into a poor prognosis. Our findings support this observation that patients with failed karyotype should be considered higher-risk patients. Together, new techniques such as MLPA should be used to overcome the technical challenges associated with cases without result after banding cytogenetics.

For the impact of cytogenetic aberrations detected by MLPA, as shown in Fig. 2, OS in 24 patients harboring abnormalities detected just by MLPA was significantly shorter compared to others. The impact of cytogenetic aberrations detected by MLPA on OS of different IPSS-R patients is also shown in Fig. 4. These results are consistent with the study of Wang [16]. All those suggest that MLPA has the potential to alter the risk stratification in MDS patients with normal or cases without result after banding cytogenetics.

\section{Conclusion}

In conclusion, MLPA can detect CNVs in a high-throughput fashion with higher resolution and can be used easily in routine diagnostics in MDS with normal karyotype or cases without result after banding cytogenetics, benefiting to the patients harboring submicroscopic deletions where informative prognostic factors underlying.

Supplementary Information The online version contains supplementary material available at https://doi.org/10.1007/s00277-021-04550-8.

Availability of data and materials Please contact the author for data requests.

Code availability Not applicable.

Author contribution J.M and A.F drafted the paper; A.F, J.M, L.X, X.A, and C.T provided patient information; J.M, H.Y, and Y.Y analyzed the 
data; H.Z, Z.Z, and X.W revised the paper; and Y.W and Z.C designed the research study and critically revised the paper. All authors approved all versions including the final version and are responsible for the accuracy and integrity of all aspects of the manuscript.

Funding This study was funded by the National Natural Science Foundation of China (grant 81870150), the National Natural Science Foundation of China (grant 81670102), and the National Natural Science Foundation of China (grant 81670104).

\section{Declarations}

Ethics approval and consent to participate The study was approved by the Medical Ethics Committee of the Tianjin Cancer Institute and Hospital. Because our study is retrospective, we collected and analyzed the data of enrolled patients by reviewing their electronic medical records. Informed consent was obtained orally from the included patients by telephone. The information of enrolled patients is anonymous. The collection, analysis, and publication of the data will not infringe enrolled patients' health, safety, and privacy.

\section{Consent for publication Not applicable.}

Competing interests The authors declare no competing interests.

Open Access This article is licensed under a Creative Commons Attribution 4.0 International License, which permits use, sharing, adaptation, distribution and reproduction in any medium or format, as long as you give appropriate credit to the original author(s) and the source, provide a link to the Creative Commons licence, and indicate if changes were made. The images or other third party material in this article are included in the article's Creative Commons licence, unless indicated otherwise in a credit line to the material. If material is not included in the article's Creative Commons licence and your intended use is not permitted by statutory regulation or exceeds the permitted use, you will need to obtain permission directly from the copyright holder. To view a copy of this licence, visit http://creativecommons.org/licenses/by/4.0/.

\section{References}

1. Banerjee T, Calvi LM, Becker MW, Liesveld JL (2019) Flaming and fanning: the spectrum of inflammatory influences in myelodysplastic syndromes. Blood Rev 36:57-69. https://doi.org/ 10.1016/j.blre.2019.04.004

2. Gadji M, Pozzo AR (2019) From cellular morphology to molecular and epigenetic anomalies of myelodysplastic syndromes. Genes Chromosom Cancer 58(7):474-483. https://doi.org/10.1002/gcc.22689

3. Haase D (2008) Cytogenetic features in myelodysplastic syndromes. Ann Hematol 87(7):515-526. https://doi.org/10.1007/s00277-0080483-y

4. Schouten JP, McElgunn CJ, Waaijer R, Zwijnenburg D, Diepvens F, Pals G (2002) Relative quantification of 40 nucleic acid sequences by multiplex ligation-dependent probe amplification. Nucleic Acids Res 30(12):57-557. https://doi.org/10.1093/nar/gnf056

5. Fabris S, Scarciolla O, Morabito F, Cifarelli RA, Dininno C, Cutrona G, Matis S, Recchia AG, Gentile M, Ciceri G, Ferrarini M, Ciancio A, Mannarella C, Neri A, Fragasso A (2011) Multiplex ligation-dependent probe amplification and fluorescence in situ hybridization to detect chromosomal abnormalities in chronic lymphocytic leukemia: a comparative study. Genes Chromosom Cancer 50(9):726-734. https://doi.org/10.1002/gcc.20894

6. den Dunnen JT, White SJ (2006) MLPA and MAPH: sensitive detection of deletions and duplications. Curr Protoc Hum Genet Chapter 7: Unit7.14 51. https://doi.org/10.1002/0471142905.hg0714s51

7. Neill NJ, Torchia BS, Bejjani BA, Shaffer LG, Ballif BC (2010) Comparative analysis of copy number detection by whole-genome BAC and oligonucleotide array CGH. Mol Cytogenet 3:11. https:// doi.org/10.1186/1755-8166-3-11

8. Xing R, Li C, Gale RP, Zhang Y, Xu Z, Qin T, Li B, Fang L, Zhang H, Pan L, Hu N, Qu S, Xiao Z (2014) Monosomal karyotype is an independent predictor of survival in patients with higher-risk myelodysplastic syndrome. Am J Hematol 89(10):163-168. https://doi.org/10.1002/ajh.23801

9. Shaffer LG, Slovak ML, Campbell LJ (2009) ISCN 2009: An international system for human cytogenetic nomenclature (2009): recommendations of the International Standing Committee on Human Cytogenetic Nomenclature. Karger, Basel

10. Greenberg PL, Tuechler H, Schanz J, Sanz G, Garcia-Manero G, Sole F (2012) Revised International Prognostic Scoring System (IPSS-R) for myelodysplastic syndromes. Blood 120:2454-2465. https://doi.org/10.1182/blood-2012-03-420489

11. Kearney L, Horsley SW (2005) Molecular cytogenetics in hematological malignancy: current technology and future prospects. Chromosoma 1 14(4):286-294. https://doi.org/10.1007/s00412-005-0002-z

12. Donahue A, Abdool A, Gaur R, Wohlgemuth JG, Yeh CH (2011) Multiplex ligation-dependent probe amplification for detection of chromosomal abnormalities in myelodysplastic syndrome and acute myeloid leukemia. Leuk Res 35(11):1477-1483. https://doi. org/10.1016/j.leukres.2011.06.019

13. Volkert S, Haferlach T, Holzwarth J, Zenger M, Kern W, Staller M, Nagata Y, Yoshida K, Ogawa S, Schnittger S, Haferlach C (2016) Array CGH identified copy number changes in $11 \%$ of 520 MDS patients with normal karyotype and uncovers prognostically relevant deletions. Leukemia 30(1): 257-260. https://doi.org/10.1038/leu.2015.257

14. Wang J, Ai X, Qin T, Xu Z, Zhang Y, Liu J, Fang L, Zhang H, Pan L, Hu N, Qu S, Cai W, Ru K, Jia Y, Huang G, Xiao Z (2017) Multiplex ligation-dependent probe amplification assay identifies additional copy number changes compared with R-band karyotype and provide more accuracy prognostic information in myelodysplastic syndromes. Oncotarget 8(1):1603-1612. https://doi.org/10.18632/oncotarget.13688

15. Watson MS (2007) Quality assurance and quality control in clinical cytogenetics. Current Protocols in Human Genetics. John Wiley, New York, pp 8.2.1-8.2.9. https://doi.org/10.1002/0471142905

16. Santos MFM, Oliveira FCAC, Kishimoto RK, Borri D, Santos FPS, Campregher PV, Silveira PAA, Hamerschlak N, CLP M, Duarte FB, Crepaldi AH, SalvinoMA VEDRP (2019) Pre-analytical parameters associated with unsuccessful karyotyping in myeloid neoplasm: a study of 421 samples. Braz J Med Biol Res 52(2):8194-8198. https://doi.org/ $10.1590 / 1414-431 \times 20188194$

17. Medeiros BC, Othus M, Estey EH, Fang M, Appelbaum FR (2014) Unsuccessful diagnostic cytogenetic analysis is a poor prognostic feature in acute myeloid leukaemia. Br J Haematol 164(2):245250. https://doi.org/10.1111/bjh.12625

18. Lazarevic V, Hörstedt AS, Johansson B, Antunovic P, Billström R, Derolf Å, Lehmann S, Möllgård L, Peterson S, Stockelberg D, Uggla B, Vennström L, Wahlin A, Höglund M, Juliusson G (2015) Failure matters: unsuccessful cytogenetics and unperformed cytogenetics are associated with a poor prognosis in a population-based series of acute myeloid leukaemia. Eur J Haematol 94(5):419-423. https://doi.org/10. 1111/ejh. 12446

Publisher's note Springer Nature remains neutral with regard to jurisdictional claims in published maps and institutional affiliations. 